\title{
Development of acute renal failure after magnetic resonance with gadopentetate
}

\begin{abstract}
Gadolinium $(\mathrm{Gd})$ based contrast materials are traditionally considered non nephrotoxic. In the past, acute renal failure (ARF) has been reported after gadolinium administration in some publications. A 65year old female patient was underwent upper abdominal MR examination. She had no history of diabetes, hypertension and kidney problems. Neither there was any history of NSAIDs nor nephrotoxic agents. During examination process, $50 \mathrm{mg}$ gadopentetate was used. Three days later, rapidly progressive anuric acute renal failure developed. In anesthesia intensive care unit, she was underwent dialysis for six sessions. Her renal biopsy was carried out and its results turned out to be acute tubular necrosis and acute intersistial nephritis. Acute renal failure improved after subsequent hemodialysis sessions. General condition and laboratory values improved, all treatment was gradually withdrawn and and the patient was discharged without any complications.
\end{abstract}

Keywords: acute renal failure, magnetic resonance, gadolinium
Volume 2 Issue 5 - 2015

\author{
Oznur Kal,' Esra Zeynep Coskunoglu, ${ }^{2}$ Ulas \\ Pinar $\mathrm{H}^{3}$ \\ 'Department of Nephrology, Baskent University, Turkey \\ ${ }^{2}$ Department of Pathology, Baskent University, Turkey \\ ${ }^{3}$ Department of Anesthesia and Intensive Care, Baskent \\ University, Turkey
}

\begin{abstract}
Correspondence: Oznur Kal, Department of Nephrology, Baskent University Medical Faculty, Konya Hospital, Turkey, Tel +905059052252, Email dr.oznurkal@yahoo.com
\end{abstract}

Received: October 07, 2015 | Published: October 23, 2015
Abbreviations: Gd, gadolinium; ARF, acute renal failure; CT, computed tomography; CIN, contrast-induced nephropathy

\section{Introduction}

MR images are superior to obtained with computed tomography (CT) scan and it has the advantage of avoiding iodinated radio contrast agents which have more overall toxicity (allergic and nonallergic reactions). Because imaging procedures with iodinated radiological contrast agents remain an important cause of acute renal failure (ARF) for hospitalized patients. The pathogenesis of contrastinduced nephropathy $(\mathrm{CIN})$ is likely to be multifactorial and the main risk factors for the development of CIN such as chronic renal insufficiency, older age, concomitant use of other nephrotoxic agents, hypotension, diabetes mellitus, and congestive heart failure are well defined. ${ }^{1}$ MR images are significantly enhanced by use of gadoliniumbased contrast agents. Gadolinium (Gd) based contrast materials are traditionally considered non nephrotoxic. But in the past, acute renal failure (ARF) has been reported after gadolinium administration in some publications. ${ }^{2} \mathrm{Gd}$ contrast-induced nephropathy has been raised over the past decade in patients with underlying kidney disease and other co-morbidities. Because hyperosmolar Gd-contrast agents are completely eliminated via glomerular filtration. Additionally Gdcontrast exposure in patients with significant kidney disease may trigger the development of nephrogenic systemic fibrosis. ${ }^{3}$ Nephrogenic systemic fibrosis is characterized by thickening and hardening of the skin with fibrotic plaques and papules and brawny hyperpigmentation in addition to possible extracutaneous manifestations. ${ }^{4}$ There were some adverse factors such as repeated MR measurements and high risk of patients for ARF. The aim of this case report was to demonstrate the fact that a patients with no risk factors for ARF may have progressive acute renal failure after undergoing even a single MR examination.

\section{Case}

A 65year old female patient, at the weight of $75 \mathrm{~kg}$ and height of $165 \mathrm{~cm}$ underwent upper abdominal MR examination with the presumptive diagnosis of mesenteric ischemia when she sought medical help for abdominal pain. She had no history of diabetes, hypertension and kidney problems. Neither there was any history of NSAIDs nor nephrotoxic agents. During examination process, $50 \mathrm{mg}$ gadopentetate $(0.66 \mathrm{mg} / \mathrm{kg})$ was used. MR revelaed no pathological findings. 3 days later, rapidly progressive anuric acute renal failure developed. Her first serum creatinine concentration was $2.4(\mathrm{mg} / \mathrm{dl})$ and GFR was $36,2\left(\mathrm{ml} / \mathrm{dk} / 1.73 \mathrm{~m}^{2}\right)$ among $0-6 \mathrm{~h}$ and second creatinine was $2.5(\mathrm{mg} / \mathrm{dl})$ and GFR was 34,3 (ml/dk/1.73m²) among 6-24h.

In anesthesia intensive care unit, she was underwent dialysis for six sessions. Renal biopsy was carried out. Biopsy results turned out to be acute tubular necrosis and acute intersistial nephritis. Anticoagulant s.c. enoxaparin 2 doses $* 4000 \mathrm{U}$ was administered to the patient. Due to developing iron deficiencey anemia, IV. iron sucrose treatment was administered three days a week. Owing to severe nausea episodes, as antiemetic metoklopramid and hydration along with nephramin (aminoacid) solution were commenced. For deep acidosis clinical picture in acute renal failure process, hemodiallyis in combination with oral plus iv sodium bicarbonate was employed. As the patient was oliguric, diuresis was attempted to be increased with furosemide as diuretic. Pantoprazol and prophylactic seftriaxon were administered. Calcium acetate was administered against phosphorus levels increasing in acute renal failure process. After polyurea phase was entered, K supplementation was given for decreased potassium levels. Uremia picture and oral intake improved, nutrition solutions and parenteral solutions were discontinued. Acute renal failure improved after subsequent hemodialysis sessions. General condition and laboratory values improved, all treatment was gradually withdrawn and the patient was discharged without any complications.

\section{Discussion}

Gd based contrast media were introduced as alternatives to iodinated media for magnetic resonance imaging. Gd based contrast media have significantly lower volumes, making them potentially less nephrotoxic. But over the past decade, there has been controversy regarding the renal safety of Gd based contrast media in patients with renal impairment. ${ }^{5}$ While Gd based contrast media were initially 
thought to be a safe alternative to iodinated radiological contrast agents for patients with chronic renal insufficiency, many clinical studies and case reports of gadolinium-associated acute kidney injury have now been recorded. Recent clinical observations report contrast induced nephrophaty after the administration of Gadolinium based contrast agents in patients with renal impairment, with an incidence of $3.5 \%-50 \%{ }^{6}$

The underlying mechanism of Gd induced renal injury is not completed understood. Some risk factors for contrast induced nephropathy have been well defined: chronic renall insufficiency, older age, concomitant use of other nephrotoxic agents, hypotension, diabetes mellitus and congestive heart failure are the main risk factors for the development of contrast-induced nephropathy. Gdcontrasts have characteristics very similar to iodinated radio contrast, in particular hyperosmolality and renal clearance entirely dependent upon glomerular filtration, nephrotoxicity was an obvious concern of both manufacturers and physicians. Animal studies demonstrate nephrotoxicity with gadolinium contrast when given in high doses $(0.6-3.0 \mathrm{mmol} / \mathrm{kg})$ to rats with normal kidney function. ${ }^{7}$

Even up until recently, the Gd-contrasts were considered relatively safe for use in patients with kidney disease, even with the high doses required for renovascular imaging, where most other imaging techniques were inadequate or were too risky such as ultrasonography or CT with iodinated contrast. Nevertheless the evidence of nephrotoxicity due to $\mathrm{Gd}$ is conflicting and review of the literature on this subject does not allow a definitive answer to the question of Gd contrast nephrotoxicity. But a typical non-vascular MRI examination employs $0.1 \mathrm{mmol} / \mathrm{kg}$ often utilizes up to $0.3 \mathrm{mmol} / \mathrm{kg}$ of Gd-contrast. And indeed in our patient high doses $(0.66 \mathrm{mg} / \mathrm{kg}) \mathrm{Gd}$ was given. As to our opinion the dose of Gd-contrast utilized appears to be importantly associated with the development of acute kidney injury.

\section{Conclusion}

Although Gd based contrast media have been traditionally considered as non-nephrotoxic, have recently been reported to be associated with acute kidney injury. In the present case, although there was no risk factor for acute renal failure, anuric renal failure developed following MR imaging, which is a source of concern. It should be borne in mind even in patients without any risk factor for ARF development, the cases should be hydrated adequately and monitorized closely as a measure against the development of ARF.

\section{Acknowledgements}

None.

\section{Conflict of interest}

The author declares no conflict of interest.

\section{References}

1. Bartholomew BA, Harjai KJ, Dukkipati S, et al. Impact of nephropathy after percutaneous coronary intervention and a method for risk stratification. Am J Cardiol. 2004;93(12):1515-1519.

2. Perazella MA. Gadolinium-Contrast Toxicity in Patients with Kidney Disease: Nephrotoxicity and Nephrogenic Systemic Fibrosis. Curr Drug Saf. 2008;3(1):67-75.

3. Perazella MA, Rodby RA. Gadolinium use in patients with kidney disease: A cause for concern. Semin Dial. 2007;20:179-185.

4. Cowper SE, Su LD, Bhawan J, et al. Nephrogenic fibrosing dermopathy. Am J Dermatopathol. 2001;23(5):383-393.

5. Penfield JG, Reilly RF. What nephrologists need to know about gadolinium. Nat Clin ract Nephrol. 2007;3(12):654-668.

6. Ledneva E, Karie S, Launay-Vacher V, et al. Renal safety of gadoliniumbased contrast media in patients with chronic renal insufficiency. Radiology. 2009;250(3):618-628.

7. Wible JH, Troup CM, Hynes MR, et al. Toxicological assessment of gadoversetamide injection (OptiMARK), a new contrastenhancement agent for use in magnetic resonance imaging. Invest Radiol. 2001;36(7):401-412. 